\title{
Posterior Central Cord syndrome following a Hyperextension injury: Case Report
}

\author{
J. G. Belen, MD, S. I. Weingarden, MD \\ Southeastern Michigan Spinal Cord Injury System, Rehabilitation Institute of \\ Detroit, 261 Mack Boulevard, Detroit, Michigan 48201, USA.
}

\section{Summary}

Throughout the spinal cord literature, a posterior central cord syndrome has been noted to be a rare occurrence. Furthermore, it has never been correlated with a specific type of injury. A patient with clinical signs consistent with a posterior central cord syndrome is discussed, and hyperextension is suggested as a possible mechanism for this type of injury.

Key words: Posterior central spinal cord syndrome; Injury mechanism; Hyperextension injury.

Spinal cord injuries may be classified according to the specific anatomical location of the injury. Hyperextension injuries in the elderly have become closely identified with a central cord syndrone, as described by Schnieder. The posterior cord syndrome, frequently mentioned in the literature as a rare occurrence, has not been correlated with a specific injury. (In fact, a literature review including a Med-line search for the posterior cord syndrome has failed to identify specific reports of this syndrome following trauma.) In one summary of injuries and their resultant neurological deficits, (Bedbrook, 1966) the posterior cord syndrome was associated with hyperextension injuries. We present here a patient with findings consistent with a posterior cord syndrome as well as an associated central cord syndrome.

\section{Case report}

A 41 year old male was riding his motorcycle and struck the rear end of a car on 23/8/83. He was thrown off the motorcycle and landed on to his back, shoulders and neck. He did not lose consciousness. He experienced immediate numbness over his trunk, arms and legs, but stated that he never lost the ability to move his legs. On arrival at the emergency room, the patient was found to have posterior neck tenderness. Deep tendon reflexes were symmetrically present and described as normal. Muscle strength testing in the arms indicated $1 / 5$ to $2 / 5$ in the deltiod and biceps and $0 / 5$ at the triceps, wrists and hand intrinsic muscles. Strength throughout the lower extremities was noted to be $2 / 5$ to $3 / 5$. Findings were identical right and left. 
Sensation to pinprick was initially absent below the C5 dermatome. Rectal tone was diminished and the bulbocavernosus reflex was absent. No findings of proprioception were noted.

The patient was started on steriods and immobilised in a Philadelphia collar. On $12 / 9 / 83$ a cervical myelogram was performed which demonstrated cervical canal stenosis. As a result, on 20/9/86 the patient underwent a C4 to C7 decompression laminectomy. After an unremarkable post-operative course, the patient was transferred to a spinal cord injury rehabilitation centre on the sixth post-operative day.

Evaluation at the time of admission to the rehabilitation centre demonstrated deltoids to be $1 / 5$, right biceps $3 / 5$, left biceps $2 / 5$ and absent triceps, wrist and hand function. Lower extremity strength was 3 to $4 / 5$ throughout. Tone was slightly increased in the lower extremities. Sensory testing demonstrated sharp/dull sensation to be intact to the C5 level, impaired at the C6 level and absent below that level. Temperature sensation was absent below C5. Proprioception was present, but decreased at the shoulder joints and absent at all other distal joints. There was neither sensation nor appreciation of bladder or bowel fullness and perianal sensation was absent.

Over the next few months, the patient showed progressive increase in strength. On repeat examination 3 months after admission, the muscle strength in the upper extremities was 3 to $4 / 5$, including the previously absent triceps, wrist flexors and extensors and hand extrinsic and intrinsic muscles. The lower extremity muscles were all in the 4 to $5 / 5$ range. Sensation improved slightly as well. Proprioception and vibration were almost normal in the upper extremities. The left leg demonstrated $20-50 \%$ accuracy of position sensation. No proprioception sensation or vibratory sensation had returned to the right lower extremity. Sharp/dull discrimination and temperature sensation were intact to C7, impaired to T4 and absent caudally. A small area of deep pressure sensation was found on the plantar aspect of the left foot.

Somatosensory evoked potentials were done at this time. No response was noted from stimulation of the right tibial nerve. Low amplitude variable responses were seen, however, from stimulation of the left tibial nerve. These findings were felt to be consistent with a relatively small number of afferent fibres conducting in the left tibial nerve.

The patient's inpatient rehabilitation program terminated on $15 / 1 / 84$. Upon discharge the patient was ambulating using visual biofeedback to compensate for absent proprioception and impaired sensory feedback. When seen in the follow-up visit 2 months later, he was able to safely ambulate at home with a walker although his gait pattern was wide based, and his foot placement hesitant. However, he did require sufficient illumination, and was unable to walk at night without lights.

\section{Discussion}

Spinal cord injuries occur through various mechanisms including flexion, with or without dislocation, compression, and hyperextension. It is likely that hyperextension is the mechanism involved when no roentgenographic abnormalities are seen. The clinical syndrome frequently associated with this injury was first described by Schneider and was termed acute cervical central cord syndrome (Schneider, 1954).

It has been theorised (Schneider, 1954) that in a hyperextension injury the cord is squeezed in an anteroposterior direction. Posteriorly, the infolding ligamentum flavum and anteriorly, a backward subluxation of the vertebral body may cause compression. There is either a rupture of the disc or fracture through the vertebral body that allows the subluxation to occur. A central hematomyelia results which produces the central cord syndrome.

The differential involvement of the arms and legs is a consequence of the somatotopic division of the lateral posterior columns of the spinal cord as 
described by Foerster in 1936 (Foerster, 1936). The central portions of the lateral spinal thalamic tracks, the spinal thalamic tracks and the posterior columns contain fibres for the neck and arms while the lateral portion parts contain the thoracic, lumbar and sacral fibres. As a result, the central haematomyelia will involve fibres of the arms more so than those of the legs. Bedbrook (1966) briefly mentions posterior cord syndrome pain and associates this with an 'extension-rotation' type of injury.

The neurological findings presented in the case reported were consistent with a posterior cord syndrome. The patient also demonstrated clinical findings compatible with a central cervical cord syndrome, as well as evidence of significant injury to the posterior tracts of the spinal cord. The loss of pain and temperature sensation indicated that the injury extended anteriorly as well. Indeed, it was only the anterior descending motor pathways that had been spared.

This case history portrayed an injury consistent with a typical type of 'hyperextension injury' that is usually seen in older individuals, with some degree of cervical canal stenosis. However, the addition of the posterior cord syndrome to the central cord syndrome made this case unique. Moreover, the damage to the posterior tracts, and the resultant loss of position sense did create special problems in the rehabilitation of this patient. The authors have subsequently seen three additional patients with a posterior cord syndrome. Specific problems in the rehabilitation of patients with this disorder will be the subject of a future report.

\section{Acknowledgement}

Supported in part by National Institute on Disability and Rehabilitation Research. Grant No. 008535196.

\section{References}

BEDBROOK GM 1966 Pathological principles in the management of spinal cord trauma. Paraplegia 4:43-56.

FOERSTER O 1936 Symptomatologie der Erkrankungen des Rueckenmarks und seiner Wurzeln. Bumke u. Foersters Handbook of Neurology 5:1-403, p. 83.

SChNeIDER RC, Cherry G, PANTEle H 1954 The syndrome of acute central cervical spinal cord injury. Fournal of Neurosurgery 11:546-66. 